\title{
ANÁLISE DA EFETIVIDADE DE PLANOS DE MANEJO PARA O \\ DESENVOLVIMENTO SUSTENTÁVEL EM PARQUES ESTADUAIS DE MATO GROSSO DO SUL
}

\section{Analysis of the effectiveness of management plans for the sustainable development in Mato Grosso do Sul State Parks}

\section{Análisis de la efectividad de los planes de gestión para el desarrollo sostenible en los parques estatales en Mato Grosso do Sul}

\author{
Jorge Souza Pinto \\ Universidade Federal de Mato Grosso do Sul \\ jorge.pinto@ufms.br \\ Celso Correia Souza \\ Universidade Anhanguera-Uniderp \\ csouza939@gmail.com \\ André Luiz Pinto \\ Universidade Federal de Mato Grosso do Sul \\ andre.pinto@ufms.br \\ Valéria Peron de Souza Pinto \\ Universidade Federal de Mato Grosso do Sul \\ vperon@gmail.com \\ Emília Alibio Oppliger \\ Universidade Anhanguera-Uniderp \\ emiliaoppliger@ hotmail.com \\ Ademir Kleber Morbeck Oliveira \\ Universidade Anhanguera-Uniderp \\ akmorbeckoliveira@gmail.com
}

Artigo enviado para publicação em 07/07/2020 e aceito em 16/09/2020

DOI: 10.12957/tamoios.2020.

\section{Resumo}

A análise e avaliação dos planos de manejo em unidades de conservação são importantes para indicar que se os objetivos que nortearam sua criação foram atingidos. Desta maneira, objetivouse o desenvolvimento de um índice para analisar a efetividade dos planos de manejo em Parques Estaduais em Mato Grosso do Sul, levando-se em consideração os planos aprovados e em fase de aplicação. Foram utilizados dados estruturados dos parques, obtidos por meio da aplicação de formulários, buscando desenvolver um índice da conformidade, em relação a sua capacidade de manutenção dos níveis de preservação e conservação. Os resultados demonstraram a fragilidade de efetivação dos planos, além de falhas em instrumentos de orientação e tomada de decisão para sua gestão. O Parque Pantanal do Rio Negro apresentou o maior Nível de Desenvolvimento Sustentável, fator decorrente de sua localização e entorno. O Parque do Prosa, a menor nota, situação também derivada de sua localização. Por outro lado, o Parque do Prosa se destaca em relação Nível de Efetividade do Desenvolvimento Sustentável com devido sua estrutura receptiva, quase inexistente nos demais. No geral, os índices obtidos apontam para perda de qualidade ambiental das áreas, sendo que tal situação pode acarretar perda de biodiversidade.

Palavras-chave: Desenvolvimento sustentável; Gestão ambiental; Unidades de conservação. 


\begin{abstract}
The analysis and evaluation of management plans in protected areas are important to indicate that the objectives that guided their creation have been achieved. In this way, the objective was to develop an index to analyze the effectiveness of management plans in State Parks in the state of Mato Grosso do Sul, taking into account the plans approved and being applied. Structured data from the parks were obtained through the application of data collection forms, seeking to develop an index of park compliance in relation to their ability to maintain levels of preservation and conservation. The results obtained demonstrated the fragility of carrying out the plans, in addition to flaws in the instruments of guidance and decision making for their management. The Pantanal State Park of Rio Negro showed the highest level of Sustainable Development, a factor related to its location and surroundings. The Prosa State Park, the lowest score, a situation also derived from its location. On the other hand, the Prosa State Park stands out in relation to the Level of Effectiveness of Sustainable Development, because due to its receptive structure, almost nonexistent in the others. In general, the indices obtained point to a loss of environmental quality in the areas, and this situation can lead to loss of biodiversity.
\end{abstract}

Keywords: Sustainable development; Environmental management; Conservation units.

\title{
Resumen
}

El análisis y evaluación de los planes de manejo en áreas protegidas son importantes para indicar que se lograron los objetivos que guiaron su creación. De esta forma, el objetivo era desarrollar un índice para analizar la efectividad de los planes de gestión en los Parques estatales en el estado de Mato Grosso do Sul, teniendo en cuenta los planes aprobados y en ejecución. Se utilizaron datos estructurados del parque, obtenidos mediante la aplicación de formularios de recolección de datos, buscando desarrollar un índice de cumplimiento del parque en relación con su capacidad para mantener niveles de conservación. Los resultados obtenidos demostraron la fragilidad de llevar a cabo los planes, además de fallas en la orientación y los instrumentos de toma de decisiones para su gestión. El Parque Estatal Pantanal de Río Negro mostró el más alto nivel de desarrollo sostenible, un factor resultante de su ubicación y entorno. El Parque Estatal Prosa, el puntaje más bajo, una situación derivada también de su ubicación. Por otro lado, el Parque Estatal Prosa se destaca en relación al Nivel de Efectividad del Desarrollo Sostenible, ya que por su estructura receptiva, casi inexistente en los demás. En general, los índices obtenidos apuntan a una pérdida de calidad ambiental en las áreas, y esta situación puede conducir a la pérdida de biodiversidad.

Palabras clave: Desarrollo sostenible; Gestión ambiental; Unidades de conservación. 


\section{Introdução}

A amplitude inerente ao tratamento do conceito da sustentabilidade é exposta pela Comissão Mundial sobre Meio Ambiente e Desenvolvimento (CMMAD, 1988), tratando-a como desenvolvimento sustentável. A comissão entende que deve haver dois conceitos-chave: (i) o conceito de necessidades básicas dos pobres de todo o mundo, que deve ser atendida como prioridade e, (ii) o conceito de limitação dos recursos naturais, que pode impedir as gerações presentes e futuras de atenderem as suas necessidades.

A sustentabilidade em Unidades de Conservação coaduna com os pressupostos de tornar convergentes os aspectos ambientais na preservação e conservação dos recursos naturais. Cabe ressaltar que o termo conservação significa proteção dos recursos naturais, com a utilização racional, garantindo sua sustentabilidade. Preservação, visa à integridade e à perenidade de algo, relacionado à proteção integral (OLIVEIRA e FERREIRA, 2019). O novo paradigma que se almeja é o convívio harmônico das atividades antropogênicas e suas cominações sociais e econômicas aos indivíduos e ao ambiente. Busca-se, nesse sentido, o estabelecimento de um estado de equilíbrio que possibilite a manutenção dos atributos quantitativos e qualitativos da biodiversidade, da conservação do ambiente e dos recursos naturais.

Considerando a utilização de índices e indicadores para avaliar a dinâmica de sistemas com relativa complexidade como o ambiente, a priori, um indicador de sustentabilidade deverá, em linhas gerais, definir ou monitorar a sustentabilidade do ambiente estudado. Aliado a isso, deve também ter capacidade de facilitar o processo de tomada de decisão, evidenciar em tempo hábil uma modificação significativa, detectar os limites entre o colapso e a capacidade de manutenção, sistematizar as informações e medir o progresso em direção à sustentabilidade (OECD, 1994; EPA, 1995; HAMMOND et al., 1995; EEA, 2000; IISD, 2013).

Ao comparar as estatísticas ambientais com as de produção e de atividades econômicas disponíveis, as ambientais ainda são frágeis em termos de diversidade de indicadores e até mesmo em relação a sua objetividade. Esta divergência em conteúdos gera dificuldades de entendimento de informações do ambiente, sendo que esta deficiência se deve a dois fatores: (i) a ainda recente consciência ambiental instalada na população mundial e, (ii) a falta de clareza quanto ao cerne da questão ambiental (BESSERMAN, 2003).

Entretanto, todos reconhecem limitações nas generalizações em relação aos índices, principalmente, pela particularidade dos elementos e indicadores utilizados em sua formatação. Mas também é percebida sua contribuição no entendimento de situações e na prospecção de cenários (CARNEIRO NETO et al., 2008; PASSOS e PIRES, 2008; MACEDO et al., 2011; SANTOS et al., 2011; FREITAS et al., 2012; OSTROVSKI e PASSOS, 2012).

Van Bellen (2004) faz um estudo de síntese do que qualifica como os índices e indicadores ambientais mais reconhecidos internacionalmente para avaliação de sustentabilidade. Tal síntese relaciona nesta qualificação os índices Ecological Footprint Method, Barometer of Sustainability e Dashboard of Sustainability, salientando que na percepção de especialistas ligados a diferentes setores da sociedade, estes índices estão entre os mais relevantes e promissores, em termos de avaliação do processo de desenvolvimento sustentável.

Veiga (2010) traz relevantes contribuições para o direcionamento da escolha de dimensões adequadas para avaliação de índices de sustentabilidade, retomando as origens da discussão da sustentabilidade pela premissa da obsolescência do crescimento econômico. Segundo o autor, ao desenvolver índices de sustentabilidade é preciso reter a 
ideia básica de medir/mensurar as várias pressões humanas sobre os ecossistemas para compará-las à sua capacidade de suporte, mas sem agregá-las, para não distorcer os resultados.

Para UNECE/OECD/EUROSTAT (2008), tratando-se de sustentabilidade, qualquer indicador monetário deve permanecer focado apenas em seus aspectos estritamente econômicos. Grande parte dos elementos que interessam, no que diz respeito ao ambiente, não tem preços definidos por mercados, assim como não há nenhuma garantia de que os preços revelem a sua importância para o bem-estar futuro. Assim, há consenso que o conjunto de indicadores deve informar sobre as variações de estoques que escoram o bem-estar humano, dando maior ênfase aos aspectos ambientais da sustentabilidade, acompanhados pelo uso de indicadores físicos bem escolhidos. De acordo com Bellen (2006), os indicadores devem ser físicos, pois a sustentabilidade é ligada em grande parte a problemas físicos, tais como a água, alimentos, florestas e poluentes. Neste ponto, o conceito proposto acolhe o "princípio da precaução", dado o estado de ignorância sobre muitos aspectos importantes da sustentabilidade do ambiente.

Veiga (2010) também acrescenta como principais diretrizes para sistematizar um bom índice de sustentabilidade, a importância de requerer um pequeno conjunto bem escolhido de indicadores, diferente dos que podem avaliar qualidade de vida e desempenho econômico, ressaltando a possibilidade de interpretá-los como variações de estoques e não de fluxos.

O desenvolvimento de índices de sustentabilidade constitui um desafio especial para os pesquisadores, pois é um processo tanto de carga científica de "produção de conhecimento" a ser disseminado quanto de política de "criação de norma" e conhecimento de limites. Estes dois componentes devem ser devidamente reconhecidos e aplicados (HAMMOND et al., 1995; OSTROVSKI e PASSOS, 2012).

Diante da diversidade de unidades de conservação e de áreas protegidas no estado de Mato Grosso do Sul, buscou-se delimitar a atenção a alguns Parques Estaduais, responsáveis proteger parte de ecossistemas, como o Cerrado e o Pantanal, constantemente ameaçados pela ação antrópica, além do Cerrado ser considerado um hotspot (FRANÇOSO et al., 2015; MYERS et al., 2015).

Entre as diferentes unidades, algumas possuem planos de manejo e entre estas, se destacam o Parque Estadual Pantanal do Rio Negro (PEPRN, coordenadas 5330'0" a $53^{\circ} 07^{\prime} 0 " \mathrm{O}$ e $18^{\circ} 13^{\prime} 20^{\prime \prime}$ a $18^{\circ} 00^{\prime} 0^{\prime \prime} \mathrm{S}$, situado entre as cidades de Aquidauana e Corumbá) e Parque Estadual Nascentes do Rio Taquari (PENRT, coordenadas 5708'30" a 56 $11^{\circ} 0^{\prime \prime} \mathrm{O}$ e $19^{\circ} 46^{\prime} 40^{\prime \prime}$ a 19²0'0" S, inserido entre as cidades de Alcinópolis e Costa Rica), ambos pertencentes à Bacia Hidrográfica do rio Paraguai. Na Bacia Hidrográfica do rio Paraná estão localizados o Parque Estadual Várzeas do Rio Ivinhema (PEVRI, coordenadas $54^{\circ} 05^{\prime} 50^{\prime \prime}$ a $53^{\circ} 00^{\prime} 30^{\prime \prime} \mathrm{O}$ e $23^{\circ} 14^{\prime} 15^{\prime \prime}$ a $22^{\circ} 37^{\prime} 30 \mathrm{~S}$, localizado entre as cidades de Jateí, Naviraí e Taquarussu) e os Parques Estaduais Matas do Segredo (PEMS,

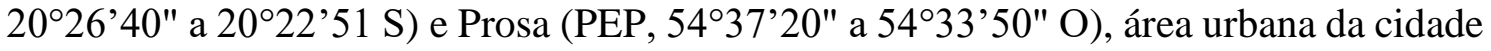
de Campo Grande (Figura 1). 


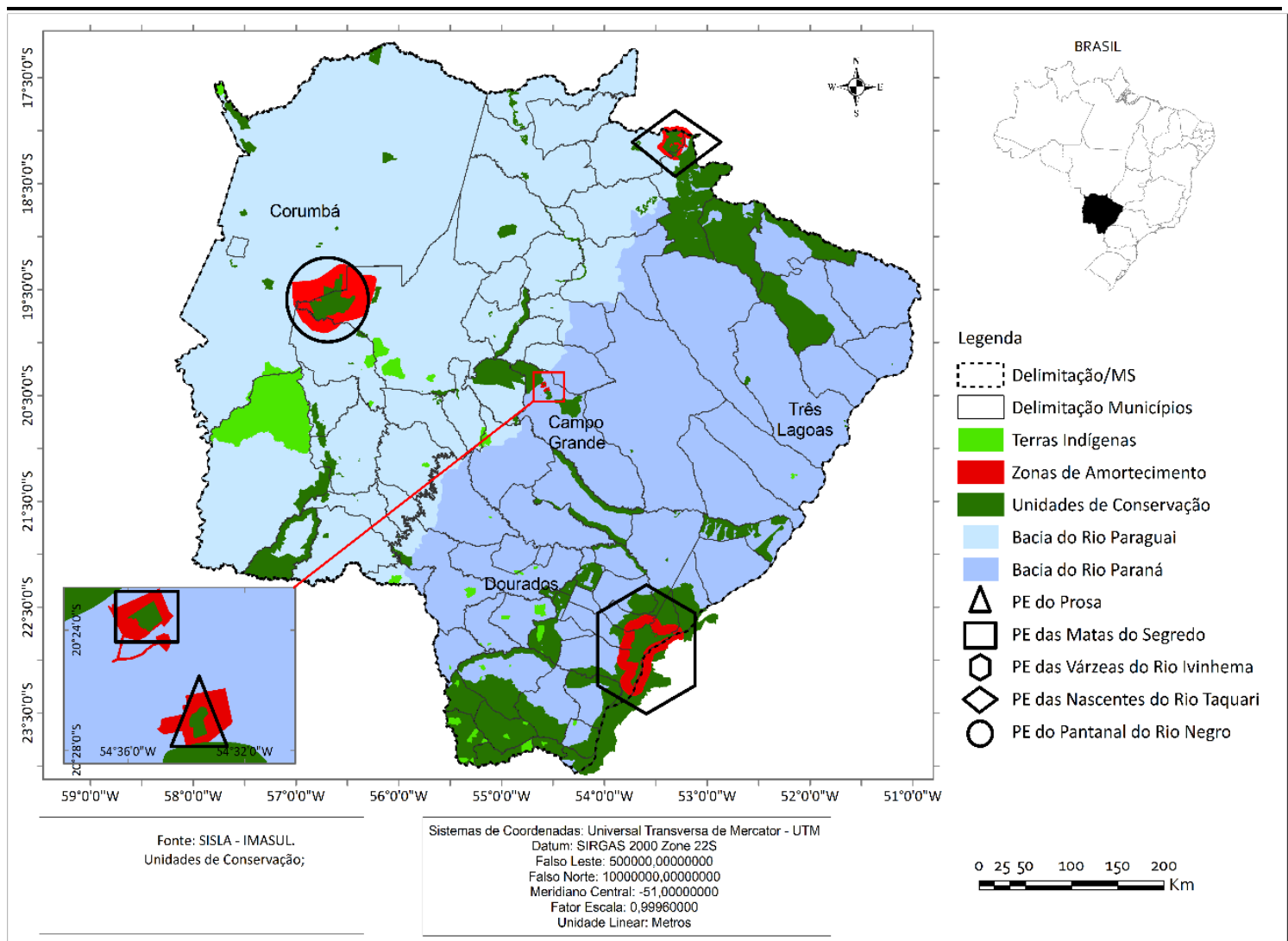

Figura 1: Parques Estaduais com planos de manejo, Mato Grosso do Sul, Brasil. Fonte: Adaptado do Sistema de Licenciamento Ambiental - Instituto de Meio Ambiente de Mato Grosso do Sul

A construção de um índice de desenvolvimento sustentável, usando a efetividade dos planos de manejo e os aspectos de cobertura vegetal e uso e ocupação da terra, gera informações que podem promover a preservação de um ambiente entendido como hotspot. Este índice se propõe a orientar a tomada de decisão a fim de direcionar esforços públicos e privados, seja por meio de Pagamento de Serviços Ambientais ou por meio da ampliação e reestruturação das políticas de Compensações Ambientais, ambas no sentido de permitir a construção de um arcabouço de desenvolvimento sustentável durável para os parques.

Objetivou-se, portanto, o desenvolvimento de um índice para analisar a efetividade dos planos de manejo em Parques Estaduais, levando-se em consideração os planos aprovados e em fase de aplicação.

\section{Material e Métodos}

Para a obtenção dos índices de Desenvolvimento Sustentável, em relação à efetividade dos planos de manejo, foram avaliadas informações destes planos e sua conciliação com a situação in loco, através de trabalhos de campo (verdade real) realizados aos Parques Estaduais.

O primeiro índice aborda o Nível de Desenvolvimento Sustentável contido na estrutura do Plano de Manejo, dividido em 23 itens com 252 questões. Por sua vez, o segundo trata do Nível de Efetividade de Desenvolvimento Sustentável dos planos de manejo com a aplicação de um formulário, o qual foi respondido pelo gestor/supervisor local, in loco, nas dependências do parque. O formulário foi dividido em 19 itens com 43 questões, baseando-se em IBAMA (2002) e abrangendo questões referentes a infraestrutura, manutenção, recursos humanos e financeiros, por exemplo. 
A classificação desenvolvida para os termômetros de efetividade de desenvolvimento sustentável nos parques foi elaborada considerando uma escala Likert de 7 pontos (Tabela 2). Foram considerados três níveis positivos e/ou de ascensão, um ponto de estagnação e outros três níveis negativos e/ou de potencial depleção, em relação as condições de desenvolvimento sustentável proporcionado pela existência e efetividade do plano de manejo, considerando as condições observadas/pesquisadas

Para calcular os índices, primeiramente pontuaram-se as questões listadas nos formulários, graduadas em uma escala Likert de 7 pontos, e cada questão um escore, calculado pela divisão da pontuação obtida por 7, ou seja, se determinada questão recebeu uma nota 5 , seu escore foi de 0,71 .

Para o cálculo do item, foi feito a soma dos escores e dividido pela quantidade de questões. Deste modo, para cada item e questão, a pontuação máxima é de 1 e a mínima de 0 . Por fim, foi feita a soma de todos os escores, dividiu-se pela quantidade de itens e obtendo-se assim, os escores dos índices.

A análise foi realizada em níveis de desenvolvimento sustentável prospectivo, sendo classificados do menor para o maior, em sete grupos: Insustentável; Declinantemente insustentável; Potencialmente insustentável; Intermediário; Potencialmente sustentável; Ascendentemente sustentável; e, Sustentável. As informações foram aplicadas sobre o conteúdo obrigatório dos planos de manejo para capacitar os Parques Estaduais ao desenvolvimento sustentável (Tabela 1).

Tabela 1: Escores dos níveis de desenvolvimento sustentável prospectivo (DSPM Desenvolvimento Sustentável do Plano de Manejo; EDSPM - Efetividade de Desenvolvimento Sustentável do Plano de Manejo)

\begin{tabular}{cc}
\hline Nível & Intervalos dos Escores de classificação \\
\hline $\mathbf{3}$ & Sustentável $(0,871 \leq \mathbf{D S P M} / \mathbf{E D S P M} \leq 1,00)$ \\
$\mathbf{2}$ & Ascendentemente Sustentável $(0,726 \leq \mathbf{D S P M} / \mathbf{E D S P M} \leq 0,870)$ \\
$\mathbf{1}$ & Potencialmente Sustentável $(0,581 \leq \mathbf{D S P M / E D S P M} \leq 0,725)$ \\
$\mathbf{0}$ & Intermediário $(0,436 \leq \mathbf{D S P M}$ /EDSPM $\leq 0,580)$ \\
$\mathbf{- 1}$ & Potencialmente Insustentável $(0,291 \leq \mathbf{D S P M} / \mathbf{E D S P M} \leq 0,435)$ \\
$\mathbf{- 2}$ & Declinantemente Insustentável $(0,146 \leq \mathbf{D S P M} / \mathbf{E D S P M} \leq 0,290)$ \\
$\mathbf{- 3}$ & Insustentável $(0,00 \leq \mathbf{D S P M} / \mathbf{E D S P M} \leq 0,145)$ \\
\hline
\end{tabular}

Para a análise dos dados, foram utilizados os seguintes testes estatísticos: (i) Intervalo de classe, por meio de um termômetro, (ii) o teste de Shapiro-Wilks, para verificar a normalidade dos escores e, (iii) a correlação de Pearson e Spearman, para verificar a correlação entre o índice Nível de Desenvolvimento Sustentável do Plano de Manejo (DSPM) e o Nível de Efetividade de Desenvolvimento Sustentável do Plano de Manejo (EDSPM) nos referidos parques (Tabela 1).

O teste de Shapiro-Wilks avalia a normalidade univariada dos dados, verificando se a variável possui ou não uma distribuição normal/regular no caso de pequenas amostras $(\mathrm{n} \leq 30)$. A hipótese nula $H_{0}$ afirma que a amostra provém de uma população normal, mas a alternativa $H_{l}$ indica que a amostra não provém de uma população normal (MAROCO, 2007).

O coeficiente de correlação de Pearson e o coeficiente de correlação de Spearman são técnicas estatísticas que medem o grau da correlação, ou seja, da associação entre duas variáveis de escala métrica. Este coeficiente, normalmente, assume apenas os valores entre -1 (quando uma variável aumenta e a outra diminui por um valor 
proporcional ou vice-versa) e +1 (quando uma variável aumenta a outra diminui proporcionalmente ou vice-versa) (FIELD, 2009).

O coeficiente de correlação de Pearson é uma estatística paramétrica e, assim, deve ser utilizada quando os dados não violarem as suposições paramétricas, ou seja, os dados possuem uma distribuição normal. Por sua vez, o coeficiente de correlação de Spearman é uma estatística não-paramétrica e, logo, deve ser usado quando os dados violarem suposições paramétricas, ou seja, os dados não possuem uma distribuição normal (FIELD, 2009). O teste de normalidade dos dados e os testes de coeficiente de correlação foram gerados com o auxílio do software estatístico Statistical Package for the Social Sciences (SPSS).

\section{Resultados e Discussão}

A construção de avaliações do cenário atual da gestão de unidades de conservação e a prospecção de cenários futuros, tendo por base seus planos de manejo e respectiva efetividade da situação de desenvolvimento sustentável, é fundamental. Esta importância é reiterada em trabalhos que avaliam a diversidade, o desenvolvimento sustentável e a gestão dos recursos naturais, pois os planos oferecem parâmetros para avaliar a evolução e/ou a não evolução de diferentes aspectos dos parques (SOUSA e SERAFINI, 2018; WHITE et al., 2019; BERNARDI et al., 2020).

Entre os parques, o que apresentou melhor Nível de Desenvolvimento Sustentável do Plano de Manejo foi o Parque Estadual do Pantanal do Rio Negro (Tabela 2). Este resultado decorre de o fato do parque fazer parte de um corredor ecológico, localizado em uma região do Pantanal circundada por várias Reservas Particulares do Patrimônio Natural (RPPN). Além disso, a região como um todo pertence à planície de inundação do rio Paraguai, o que torna a região, em termos de ocupação, mais complexa, limitando os impactos antrópicos.

A localização do parque, parcialmente protegido por outras áreas de conservação, permite que ele atue como um centro de proteção e dispersor de espécies ameaçadas, mesmo aquelas consideradas topo de cadeia, como a onça pintada, que necessita de grandes áreas preservadas para sua manutenção. A existência de diferentes Unidades de Conservação, próximas umas às outras, criando grandes áreas protegidas, é um mecanismo eficaz de proteção ambiental, prevista na Lei 9.985, que institui o Sistema Nacional de Unidade de Conservação (BRASIL, 2000).

Lima e Franco (2014), ao estudarem as RPPNs localizadas em torno do Parque Nacional da Chapada dos Veadeiros, Goiás, indicaram que as mesmas, por apresentarem restrições de uso semelhantes às unidades de conservação, podem promover a conservação de seus habitats sem grandes intervenções humanas, em parceria com a comunidade local.

O plano de manejo do PEPRN enfatiza elementos que ponderam a situação de importância do parque no contexto de bioma Cerrado e aborda o contexto dele como regulador de importante ciclo de biodiversidade do Pantanal. Entretanto, possui insuficiências significativas no desempenho de sua efetivação, comprometendo o plano como estrutura de tomada de decisões. 
Tabela 2: Escores dos níveis de desenvolvimento sustentável prospectivo em Parques Estaduais com Plano de Manejo, Mato Grosso do Sul, Brasil

\begin{tabular}{lcc}
\hline Parques Estaduais & $\begin{array}{c}\text { Nível de Desenvolvimento } \\
\text { Sustentável }\end{array}$ & $\begin{array}{c}\text { Nível de Efetividade do } \\
\text { Desenvolvimento } \\
\text { Sustentável }\end{array}$ \\
\hline Pantanal do Rio Negro & 0,611 & 0,247 \\
Nascentes do Rio Taquari & 0,572 & 0,274 \\
Várzeas do Rio Ivinhema & 0,549 & 0,254 \\
Matas do Segredo & 0,537 & 0,275 \\
Prosa & 0,429 & 0,386 \\
\hline
\end{tabular}

Em sequência, os parques melhores classificados são Nascentes do Rio Taquari e Várzeas do Rio Ivinhema. Ambos estão cercados por áreas de intensa atividade agropecuária, além de pequenas e médias cidades, sofrendo os impactos de invasões, retirada de madeira, caça e pesca ilegal e queimadas. Desta maneira, sua área e seu entorno possuem uma escala maior de pressão antrópica, o que vem a impactar negativamente os locais.

Os parques com menor pontuação, Matas do Segredo e Prosa, estão localizados em áreas urbanas, ou seja, sujeitos a todo tipo de pressão antrópica, com melhor situação para o PEMS, pois, apesar de ser parcialmente cercado por áreas residenciais, ainda possui ligação com outras áreas não urbanizadas, tais como fazendas. Porém, a expansão contínua da área urbana, sem levar em consideração a Lei 9.985 e seus mecanismos eficazes de proteção ambiental, como zonas de amortecimento, também levarão, em pouco tempo, à perda de qualidade ambiental.

Os índices obtidos, em relação ao desenvolvimento sustentável prospectivo dos planos de manejo, indicam questões estruturais inadequadas para o processo de gestão de todos os parques. Desta maneira, não é assegurado sua manutenção, dentro de seus objetivos primordiais de preservação e conservação do ambiente.

$\mathrm{Na}$ avaliação dos planos de manejo, os aspectos que poderiam tornar a condução das decisões de recomposição de cobertura vegetal, convergências de ações com as comunidades municipais e entidades privadas locais aos parques, visando à conservação do ambiente, são superficiais. E o fator que mais inibe a capacidade dos planos é o de que as previsões orçamentárias e financeiras, isto é, as origens de recursos, planos de investimentos, políticas de divulgação, visitação e aparelhamento dos parques, não estão fundamentados, passando a impressão de que as Unidades de Conservação estão invisíveis ao orçamento do Estado, o que acarreta as consequências de instabilidade operacional. Estes aspectos ficaram latentes nas exposições dos gestores/supervisores, que citaram situações em que a falta de recursos é latente. Por exemplo, a necessidade de fazer um aceiro para proteger a área de queimadas ou a recomposição de cerca ou pontes, cuja providência muitas vezes depende de doações dos municípios do entorno para sua viabilização.

Desta maneira, não existindo uma fonte de recursos definida, os planos apresentam limitações em estabelecerem objetivos consistentes, com prazos de execução e aporte de recursos que permitam a condução de ações voltadas à continuidade e melhoramento das unidades.

Uma dualidade pode ser observada em relação ao PEP, que apresentou o menor escore, mas também é o que tem melhor estrutura para visitação. Porém, dentre os planos de manejo, demonstra mais limitações em sua configuração para criar condições de conformidade às instalações e ao ambiente do parque. Neste ponto destacam-se como aspectos mais comprometedores a não previsão de adequabilidade de trilhas, dotação de 
pessoal, construções em áreas que deveriam ser de preservação, como próximo às nascentes, além dos aspectos orçamentários, que como nos demais, é indefinido no plano.

Por outro lado, o PEP apresentou o maior Nível de Efetividade do Desenvolvimento Sustentável do Plano de Manejo (Tabela 2), pois é o único que possui um receptivo, ou seja, uma estrutura de visitação com agendamentos, controle e fiscalização.

Além disso, por ter uma área menor, apresenta maior cobertura vegetal continua, embora, sob muitos aspectos, sofrendo depleções causadas pelas áreas urbanas circunvizinhas, pelo isolamento de flora e fauna, que não permite a manutenção de sua qualidade ecológica e, principalmente, pela ação antrópica, tais como: poluição (lixo), extrativismo de plantas com fins medicinais, caça de pequenos animais e queimadas em determinados locais do parque. Embora a Lei 9.985 preveja zona de amortecimento e corredor ecológico, tal situação não pode ser observada, o que implica o mau uso dos recursos do seu entorno.

As principais circunstâncias que levaram a indicadores menores nos parques PEPRN, PENRT, PEVRI, PEMS e PEP dizem respeito a não efetividade de operação das unidades para visitação e educação ambiental, à frágil situação de legalização fundiária que impede investimentos e, por fim, à ausência de projetos mais relevantes para aparelhar o seu funcionamento.

Outros fatores que ressaltam os acentuados riscos de degradação e influenciam o potencial colapso do ambiente nestes parques são: a não dotação de pessoal capacitado e especializado em quantidade condizente com as necessidades de gestão dos parques, assim como a suscetibilidade, as pressões de atividades antrópicas agropecuárias e agrícolas em áreas do entorno e interior dos parques, além da urbanização acelerada no entorno do PEMS e PEP, não respeitando a Lei 9.985 (zona de amortecimento e corredor ecológico). Kudo et al. (2016) escrevem que são diversos os fatores que colocam em risco estes espaços, como a especulação imobiliária, crescimento urbano desordenado e a ocupação irregular do solo, mas mesmo impactos, são um recurso ambiental imprescindível para a qualidade de vida das cidades.

A expectativa é que os planos de manejo possam prover bases fundamentadas de gestão das Unidades de Conservação, com prazos de execução de projetos e fontes/quantidades de recursos e investimentos, que o pessoal envolvido na gestão tenha equipe e formação dentro dos aspectos de manejo planejados. Porém o que se observa é que isto não ocorre da maneira que deveria.

O turismo em áreas naturais é uma importante ferramenta para a conservação da biodiversidade e desenvolvimento das atividades de Educação Ambiental, assim como um recurso que pode ajudar na manutenção das Unidades de Conservação.

Entretanto, para que isso ocorra, é necessário que haja estrutura adequada que permita a visitação sem causar impactos, como receptivo capacitado, trilhas suspensas (preferencialmente) e sinalização, por exemplo, além de um produto turístico formatado, de qualidade e atrativo.

Este tipo de turismo, aliado à educação ambiental, representa uma forma de alcançar o desenvolvimento sustentável (NASCIMENTO et al., 2018), pois permite a aproximação entre o ser humano e o ambiente natural, sendo uma atividade educativa que pode aproveitar a situação de contato com a natureza para compreender a importância de sua conservação.

Deste modo, verificar que nenhum parque apresentou um escore acima de $0,5 \mathrm{em}$ relação ao nível de efetividade dos planos de manejo (Tabela 2), em relação ao processo de desenvolvimento sustentável, é decepcionante. Em outras palavras, nenhum deles apresentou boa efetividade no desenvolvimento sustentável. 
Tal fato é preocupante, uma vez que a importância dos parques reside na preservação e conservação do ambiente, bem como nos fatores relacionados às amenidades que pode proporcionar, a partir da possibilidade de visitação, preservação da paisagem e dos recursos naturais e de educação ambiental.

Como já pontuado, os parques sofrem uma série de problemas, tais como restrições orçamentárias e falta de pessoal para realizar seu monitoramento e gestão, além da infraestrutura ser insuficiente, como observado em vários locais. Com exceção da infraestrutura observada no Parque Matas do Segredo e Parque do Prosa (área urbana), as estruturas físicas encontradas nos demais parques ainda são embrionárias. Normalmente compreendem apenas uma casa, cercas, pontes e estradas - construídas pelos antigos proprietários da área, ou seja, anteriores a criação do parque. As velhas sedes acabam operando mais como ponto de apoio do que propriamente, um receptivo de um parque.

Além desta situação, não ocorreu a regularização fundiária integral da área no Parque Nascentes do Rio Taquari e Parque Pantanal do Rio Negro. Porém no entorno de todos os parques é comum a não observância das normas legais, em relação as zonas de amortecimento, que geram potenciais pressões sobre a conservação das unidades de conservação. Ou seja, a tendência atual é uma pressão cada vez maior sobre os parques.

Portanto, a efetividade dos planos de manejo é superficial. Assim, a almejada competência para proporcionar sua adequada gestão passa a sucumbir por ausência de aporte adequado de recursos, que lhes possibilitem realizar uma gestão com condições de promover o desenvolvimento de maneira sustentável.

Ressalte-se, ainda, o esforço identificado dos gestores dos PEPRN, PENRT, PEVRI, PEP e PEMS, que na ausência de condições adequadas, constroem parcerias com os municípios onde se localizam e viabilizam maneiras de manter alguns aspectos necessários de manutenção e preservação.

Os principais resultados dessas parcerias são reformas em pontes, aceiros, combate à incêndios, fiscalizações e ajuda de custo para suplementos pontuais, combustíveis, pessoal terceirizado, entre outras situações necessárias ao dia a dia das unidades.

Estas ações indicam um estágio ainda embrionário de reciprocidade dos municípios no desafio de conservação e preservação das áreas dos Parques Estaduais. Porém o poder público estadual, que deveria capitanear a condução de tais atividades, trabalha aquém de suas responsabilidades e potencial.

Os dados obtidos também permitem construir uma classificação em forma de termômetro de desenvolvimento sustentável prospectivo, desenvolvido através da escala Likert (Tabela 2), com níveis positivos, de estagnação e/ou negativos. O primeiro termômetro mostra a capacidade do plano de manejo em estruturar, projetar e atender em conformidade a determinados aspectos, necessários à divulgação, gestão, identificação, visitação, fiscalização e preservação, além da recuperação de recursos degradados e redução das pressões ambientais.

Já o segundo demonstra a efetividade da capacidade do plano de manejo, obtido em visitas realizadas aos parques (Figura 2), englobando os mesmos tópicos. 


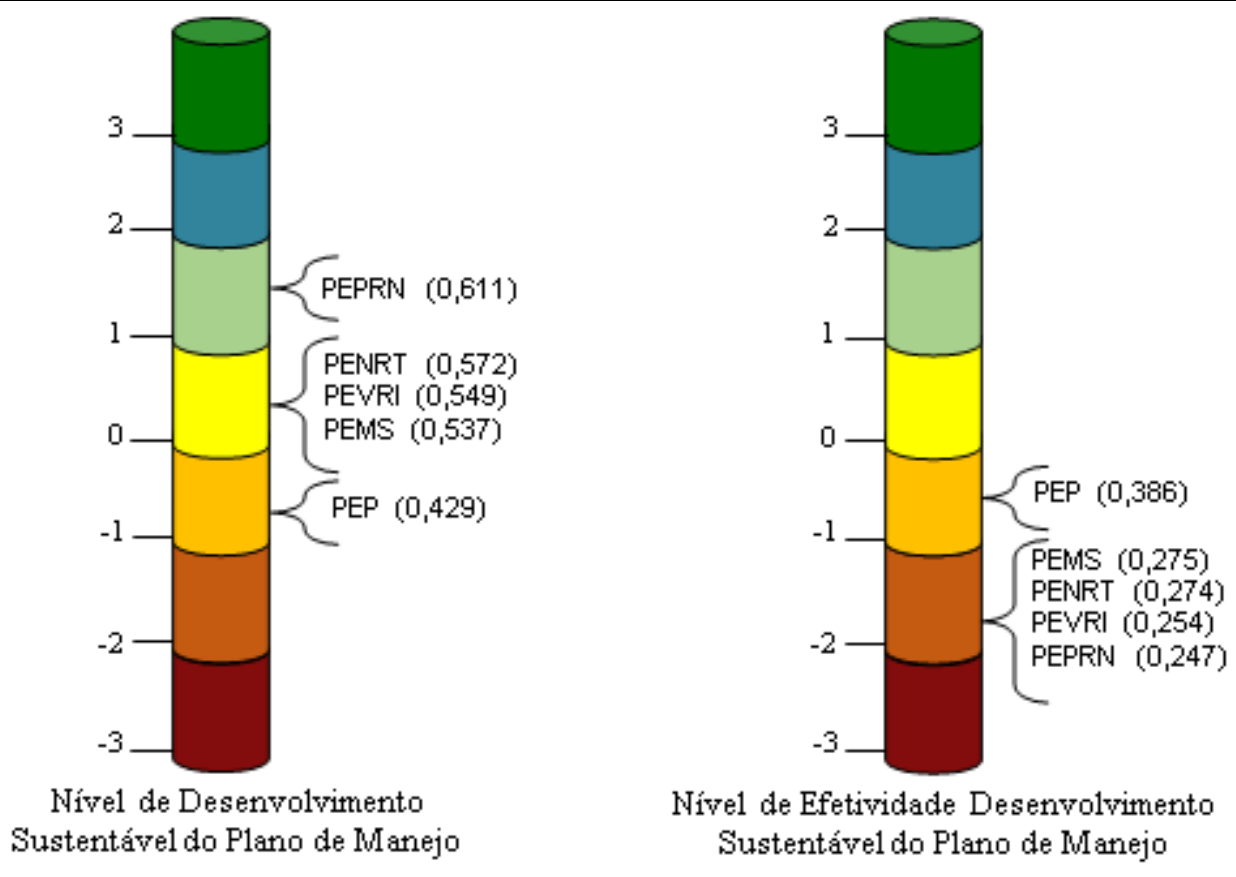

Figura 2: Termômetro de efetividade de desenvolvimento sustentável nos Parques Estaduais com planos de manejo, Mato Grosso do Sul, Brasil (3= Sustentável; 2= Ascendentemente Sustentável; $1=$ Potencialmente Sustentável; 0= Intermediário; $-1=$ Potencialmente Insustentável; -2= Declinantemente Insustentável; e, -3= Insustentável).

Quanto ao Nível de Desenvolvimento Sustentável, verifica-se que três parques PENRT, PEVRI e PEMS - ficaram na zona intermediária; como potencialmente insustentável aparece o PEP e como potencialmente sustentável, o PEPRN, sendo que em ambos, a aplicação da Lei 9.985 (ou sua falta de aplicação) estejam diretamente ligados a sua atual situação.

Verifica-se no estudo que nenhum parque foi sustentável quanto ao Nível de Desenvolvimento Sustentável, identificando-se com o resultado que a elaboração e apresentação dos planos de manejo não contemplam a estrutura que permita estabelecer paradigmas de gestão que possibilitem reunir objetivamente informações para tomadas de decisão necessárias ao manejo dos parques.

Como já pontuado, um exemplo é a omissão de previsão e definição orçamentária, o que já estabelece graves limitações na execução dos planos.

Por sua vez, em relação ao Nível de Efetividade Desenvolvimento Sustentável, todos os parques ficaram abaixo da zona intermediária, sendo um Potencialmente Insustentável (PEP) e os demais, Declinantemente Insustentável.

Tais resultados evidenciam que os parques sofrem severas restrições estruturais para colocarem em prática seus planos de manejo. A análise dados físicos de cobertura vegetal e de uso e ocupação da terra indicam que parte dos parques ainda apresentam significativa capacidade de resiliência (PENRT, PEPRN e PEVRI), por ainda reunirem uma biodiversidade representativa, em ambientes com certo nível de preservação. Porém, essas características se encontram ameaçadas devido a falta de efetivação do plano.

Já os PEMS e PEP, por estarem em uma região urbana, apresentam as mesmas demandas de preservação e conservação, em particular por abrigarem nascentes dos córregos Segredo e Prosa, principais recursos hídricos da cidade; porém apresentam biodiversidade mais exposta ao risco de depleção pelas atividades antrópicas relacionadas a expansão imobiliária, além de outros pontos já citados. 
É importante também ressaltar que os locais, que poderiam ser uma fonte de recursos financeiros de lazer para a população, em sua maioria, são praticamente abandonados pelo poder público.

Em relação aos parques urbanos, estes também apresentam diferentes funções e são responsáveis por serviços ecossistêmicos, tais como a manutenção das condições microclimáticas confortáveis à população e a minimização das condições atmosféricas críticas, como a poluição atmosférica (GAUDERETO et al., 2018). Também podem propiciar benefícios sociais e econômicos, tais como a satisfação de usuários através do uso de áreas verdes (BENAKOUCHE, 1994; XAVIER et al., 2018).

A utilização destes parques também pode refletir em um ingresso de turistas em suas áreas, o que pode ser importante para as empresas que trabalham neste setor, pois as unidades de conservação são consideradas possíveis atrativos turísticos que despertam o interesse de um público sensível ao ambiente.

O desenvolvimento do ecoturismo pode minimizar insatisfações e conflitos existentes entre as comunidades locais devido à restrição de seu uso, evitando (ou diminuindo) os impactos antrópicos causados pelos moradores do entorno. Além disso, os projetos de educação ambiental, que constam nos planos de manejo, poderiam sensibilizar parte da população que ainda não compreende a importância da conservação (MATHEUS; RAIMUNDO, 2017).

É possível avaliar os dados do Nível de Desenvolvimento Sustentável dos planos de manejo quanto à sua distribuição e foi percebido que eles possuem uma distribuição normal dos dados, pois sua estatística não foi significativa.

Já os dados do Nível de Efetividade Desenvolvimento Sustentável não apresentaram uma distribuição normal, uma vez que sua estatística foi significativa (Tabela 3).

Tabela 3: Nível de Desenvolvimento Sustentável dos planos de manejo e do Nível de Efetividade Desenvolvimento Sustentável nos Parques Estaduais com Plano de Manejo, Mato Grosso do Sul, Brasil

\section{Variáveis}

Nível de Desenvolvimento Sustentável Nível de Efetividade Desenvolvimento Sustentável gl: grau de liberdade; p: significância

\section{Shapiro-Wilk}

Estatística Gl. p.

$0,906 \quad 5 \quad 0,445$

$0,741 \quad 5 \quad 0,024$

Visto que uma das variáveis apresentou uma distribuição normal e a outra não, optou-se por fazer tanto a correlação de Pearson quanto a de Spearman (Tabela 4).

O Nível de Desenvolvimento Sustentável do Plano de Manejo (Tabela 4) está altamente correlacionado, de maneira negativa, com o Nível de Efetividade Desenvolvimento Sustentável, com um coeficiente de Pearson $\mathrm{r}=-0,943$ e Spearman $\mathrm{r}=$ $-0,900$, os quais são significativos ao nível de $5 \%$.

Tabela 4: Resultados das correlações de Pearson e a de Spearman nos Parques Estaduais com Plano de Manejo, Mato Grosso do Sul, Brasil

\begin{tabular}{ccc}
\hline & & $\begin{array}{c}\text { Nível de Efetividade de } \\
\text { Desenvolvimento Sustentável }\end{array}$ \\
\hline $\begin{array}{c}\text { Nível de Desenvolvimento } \\
\text { Sustentável }\end{array}$ & Correlação de Pearson & $-0,943$ \\
& p. & $0,016^{*}$ \\
*A correlação é significativa no nível $0,05$. & p. & $-0,900$ \\
\hline
\end{tabular}


Deste modo, verifica-se que, à medida que os níveis de desenvolvimento sustentável dos planos de manejo dos parques aumentam, sua efetividade é reduzida pela ausência da conformidade aos aspectos de divulgação, gestão, identificação, visitação, fiscalização, investimentos e preservação, com recuperação dos recursos naturais em áreas afetadas pelas ações antrópicas. Observa-se que a correlação não sugere, necessariamente, uma relação de causa e efeito, mas de associação entre as variáveis.

Os escores de desenvolvimento sustentável fomentam a necessidade de que as Unidades de Conservação sejam entendidas como entidades, que necessitam implantar sistemas de gestão. Isto vai possibilitar a melhor manutenção dos recursos naturais.

Os planos de manejo, que poderiam ser o apoio instrumental para as ações que conduziriam a gestão dos parques, para poder dotá-los de capacidade de receber visitação, servirem como ambiente de pesquisa e educação ambiental, além de serem alicerce de conservação, se relegam ao papel de serem instrumentos não efetivos, desatualizados e sem o respaldo estatal devido.

Medeiros e Pereira (2011), avaliando planos de parques nacionais do Rio de Janeiro, já alertavam que não existem trabalhos na literatura acadêmica ou relatórios institucionais, que tratem da efetividade das ações propostas nos planos. Apesar de estarem previstas no roteiro de criação, as avaliações de efetivação não estão disponíveis, indicando que não existe ainda uma correlação entre a criação dos planos e sua implantação (IBAMA, 2002).

Os índices de desenvolvimento sustentável sinalizam que determinadas ações precisam ser efetivadas, impedindo a contínua perda de qualidade dos recursos naturais. Estas ações estão ligadas a adoção de políticas públicas e medidas administrativas que alterem e efetivem a gestão dos parques. Se não forem adotados mecanismos que, efetivamente, promovam a conservação e recuperação do ambiente e implementação dos planos de manejos, sua qualidade ambiental já declinante poderá, a curto ou médio prazo, levar a perdas que dificilmente serão mitigadas.

\section{Conclusões}

Os dados obtidos indicaram a não conformidade dos planos de manejo em estabelecer diretrizes orçamentárias e financeiras para as atividades relacionadas aos Parques Estaduais, sendo um dos principais impedimentos da consolidação e operacionalização das unidades. Estas situações, aliadas a não conformidade fundiária, impedem ações efetivas que levem ao total aproveitamento do potencial de tais áreas.

Os planos ainda não se mostraram capazes de reunir o planejamento estratégico e operacional, o que promoveria condições para que os parques alcançassem sistemas de gestão eficazes para a conservação do ambiente. Portanto, a condução de sua gestão ocorre a partir de ações isoladas dos responsáveis pelas áreas.

Apesar dos problemas comuns a todos os parques, as análises realizadas indicaram que o Parque Estadual Pantanal do Rio Negro apresentou o maior Nível de Desenvolvimento Sustentável, fator decorrente de seu isolamento geográfico e a presença de RPPNs em seu entorno. O Parque Estadual do Prosa recebeu a menor nota também devido a sua localização, pois está isolado pelo ambiente urbano. Por outro lado, o Parque Estadual do Prosa, outro parque urbano, se destaca em relação ao Nível de Efetividade do Desenvolvimento Sustentável, pois sua estrutura receptiva é a adequada ao uso coletivo, situação quase inexistente nos demais parques.

Os resultados sinalizam a necessidade de que as políticas estaduais de preservação do ambiente mudem do enfoque teórico para ações mais contundentes, a fim de assegurar 
condições suficientes para que o ambiente receba os devidos investimentos para preservação.

\section{Agradecimentos}

Ao IMASUL - Instituto de Meio Ambiente de Mato Grosso do Sul, gerência de Unidades de Conservação, pelas informações e apoio para a realização do estudo. À CAPES (Coordenação de Aperfeiçoamento de Pessoal de Nível Superior) e ao CNPq (Conselho Nacional de Desenvolvimento Científico e Tecnológico), pelas bolsas de estudos concedidas (pós-graduação e Produtividade em Pesquisa). A Universidade pela infraestrutura oferecida.

\section{Referências Bibliográficas}

BELLEN, H. M. van. Desenvolvimento sustentável: uma descrição das principais ferramentas de avaliação. Ambiente \& Sociedade, Campinas, v. 7, n. 1, p. 67-88, 2004.

BELLEN, H. M. van. Indicadores de sustentabilidade: uma análise comparativa. 2.ed. Rio de Janeiro: FGV, 2006. 253p.

BENAKOUCHE, R. Avaliação monetária do meio ambiente. São Paulo: Makron Books, 1994. 198p.

BERNARDI, I.; SILVA, L. R.; FALCO, P. B.; PIRES, J. S. R.; SANTOS, A. C. A. Análise comparativa das ferramentas de gestão: Plano de Manejo da APA Itupararanga e os Planos Diretores Municipais. Sociedade \& Natureza, Uberlândia, v. 32, p. 75-91, 2020.

BESSERMAN, S. Indicadores. In: TRIGUEIRO, A. (Org.). Meio ambiente no século 21: 21 especialistas falam da questão ambiental nas suas áreas de conhecimento. Rio de Janeiro: Sextante, 2003. p. 90-95.

BRASIL. Lei do Sistema Nacional de Unidades de Conservação da Natureza - SNUC. Lei de $n^{\circ}$ 9.985 de 18 de julho de 2000, regulamentada pelo Decreto $n^{\circ}$ 4.340/2002. Regulamenta o art. 225, $\S 1$ o, incisos I, II, III e VII da Constituição Federal, institui o Sistema Nacional de Unidades de Conservação da Natureza e dá outras providências - Publicação DOU, de 19/07/2000 - Brasília. 2000.

CARNEIRO NETO, J. A.; ANDRADE, E. M.; ROSA, M. F.; MOTA, F. S. B.; LOPES, J. F. B. Índice de sustentabilidade ambiental para o perímetro irrigado Ayres de Souza. Ciência e Agrotecnologia, Lavras, v. 32, n. 4, p. 1272-1279, 2008.

CMMAD. Comissão Mundial Sobre Meio Ambiente e Desenvolvimento. Nosso futuro comum. Rio de Janeiro: Fundação Getúlio Vargas, 1988. 430p.

EEA. European Environment Agency. Questions to be answered by a state-of-the environment report. Copenhagen: European Environment Agency, 2000. Disponível em: <http://www.eea.eu.int> Acesso em: 12 jan. 2017.

EPA. United States Environmental Protection Agency. A conceptual framework to support development and use of environmental information in decision-making. 1995. Disponível em: <http://www.epa.gov/indicator/ frame/contents.html> Acesso em: 12 jan. 2017.

FIELD, A. Descobrindo a estatística usando o SPSS. 2.ed. Porto Alegre: Artmed, 2009. 688p. 
FRANÇOSO, R.; BRANDÃO, R.; NOGUEIRA, C. C.; SALMONA, Y.; MACHADO, R. B.; COLLI, G. R. Habitat loss and the effectiveness of protected areas in the Cerrado biodiversity hotspot. Natureza \& Conservação, v. 13, n. 1, p. 35-40, 2015.

FREITAS, R. C. M.; NUNES, L. S.; NÉLSIS, C. M. A crítica marxista ao desenvolvimento (in) sustentável. Revista Katálysis, Florianópolis, v. 15, n. 1, p. 41-51, 2012.

GAUDERETO, G. L.; GALLARDO, A. L. C. F.; FERREIRA, M. L.; NASCIMENTO, A. P. B.; MANTOVANI, W. Avaliação de serviços ecossistêmicos na gestão de áreas verdes urbanas: promovendo cidades saudáveis e sustentáveis. Ambiente e Sociedade, São Paulo, v. 21, p. 1-20, 2018.

HAMMOND, A.; ADRIAANSE, A.; RODENBURG, E.; BRYANT, D.; WOODWARD, R. Environmental indicators: a systematic approach to measuring and reporting on environmental policy performance in the context of sustainable development. Washington: World Resources Institute, 1995. 43p.

IBAMA. Roteiro Metodológico de Planejamento - Parques Nacionais, Reservas Biológicas e Estações Ecológicas. Brasília: Edições IBAMA, 2002. 136p.

IISD. International Institute for Sustainable Development. The dashboard of sustainability. Canada: IISD, 2013. Disponível em: < http://iisd1.iisd.ca/cgsdi/.htm> Acesso em: 15 dez. 2014.

KUDO, S. A.; PEREIRA, H. S.; SILVA, S. C. P. A proteção jurídica dos fragmentos florestais urbanos: um estudo da paisagem e da legislação ambiental e urbanística da cidade de Manaus. Desenvolvimento e Meio Ambiente, Curitiba, v. 38, p. 521-540, 2016.

LIMA, P. C. A.; FRANCO, J. L. A. As RPPNs como Estratégia para a Conservação da Biodiversidade: O Caso da Chapada dos Veadeiros. Sociedade \& Natureza, Uberlândia, v. 26, p. 113-125, 2014.

MACEDO, M. A. S.; FERREIRA, A. F. R.; CÍPOLA, F. C. Análise do nível de sustentabilidade das unidades federativas do Brasil e das Capitais: um estudo sob as perspectivas econômica, social e ambiental. Revista de Gestão Social e Ambiental - RGSA, São Paulo, v. 5, n. 3, p. 73-89, 2011.

MAROCO, J. Análise Estatística com Utilização do SPSS. 3ed. São Paulo: Edições Sílabo, 2007. 824 p.

MATHEUS, F. S.; RAIMUNDO, S. Os resultados das políticas públicas de ecoturismo em Unidades de Conservação no Brasil e no Canadá. Revista Brasileira de Pesquisa em Turismo, São Paulo, v. 11, n. 3, p. 454-479, 2017.

MEDEIROS, R.; PEREIRA, F. S. Evolução e implementação dos planos de manejo em parques nacionais no estado do Rio de Janeiro. Revista Árvore, Viçosa, v. 35, n. 2, p. 279-288, 2011.

MYERS, N.; MITTERMEIER, R. A.; MITTERMEIER, C. G.; FONSECA, G. A.; KENT, J. Biodiversity hotspots for conservation priorities. Nature, v. 403, n. 6772, p. 853-858, 2000.

NASCIMENTO, H. H. O.; MOREIRA, A. S.; SILVA, J. R. M.; ALMEIDA, N. G. Educação ambiental em ação: o papel do ecoturismo em prol da sustentabilidade das unidades de conservação. REDE - Revista Eletrônica do PRODEMA, Fortaleza, v. 12, n. 3, p. 62-68, 2018.

OECD. Environmental Indicators. Indicateurs d'environnement. OECD Core Set, Corps central de l'OECD. Paris: Organization for Economic Co-operation and Development, 1994. 155p. 
OLIVEIRA, I. C. G.; FERREIRA, A. J. Gestão e Planejamento Ambiental: Desenvolvimento Sustentável, Conservação e Preservação. Revista Eletrônica de Ciências Humanas, Saúde e Tecnologia, Uruaçu, v. 6 n. 2, p. 33-45, 2017.

OSTROVSKI, D.; PASSOS, M. M. Indicadores do desenvolvimento sustentável. Revista Eletrônica Científica Inovação e Tecnologia, Curitiba, v. 1, n. 5, p. 50-56, 2012.

PASSOS, H. D. B.; PIRES, M. M. Indicadores ambientais para avaliação de agroecossistemas. Informe Gepec, Toledo, v. 12, n.1, p. 1-15, 2008.

SANTOS, E. S.; MOTA, S.; SANTOS, A. B.; MONTEIRO, C. A. B.; FONTENELE, R. M. M. Avaliação da sustentabilidade ambiental do uso de esgoto doméstico tratado na piscicultura. Revista Engenharia Sanitária e Ambiental, Rio de Janeiro, v. 16, n. 1, p. 45-54, 2011.

SOUSA, E. E.; SERAFINI, T. Z. Panorama das Unidades de Conservação na zona costeira e marinha do estado de São Paulo. Desenvolvimento e Meio Ambiente, Curitiba, v. 44 (Edição especial: X Encontro Nacional de Gerenciamento Costeiro), p. 360-377, 2018.

UNECE/OECD/EUROSTAT. Report on measuring sustainable development: statistics for sustainable development, commonalities between current practice and theory. Paris: Working Paper, ECE/CES2008/29, 2008. 115p.

VEIGA, J. E. Indicadores de sustentabilidade. Estudos Avançados, São Paulo, v. 24, n. 68, p. 39$52,2010$.

WHITE, E. K.; COSTEllOE, J.; PETERSON, T. J.; WESTERN, A. W.; CARRARA, E. Do groundwater management plans work? Modelling the effectiveness of groundwater management scenarios. Hydrogeology Journal, Springer, v. 27, p. 2447-2470, 2019.

XAVIER, F. B.; FELIPE, J.; ARANA, A. R. A. O parque verde urbano: características do uso através de observação sistemática. Revista Brasileira de Gestão Urbana, Curitiba, v. 10, n. 1, p. 82-95, 2018. 